DOI: $10.30972 /$ eitt.704775

\title{
Presente, pasado y futuro de los materiales. Señal de la innovación para el desarrollo
}

\author{
Rada, Geraldine; Titiosky, Valeria; Esp. Cuevas, Alicia; \\ Mgter. Corvalán, Rubén(*)
}

\section{Resumen}

Históricamente, el desarrollo y evolución de las sociedades ha estado relacionada con la capacidad de sus miembros para producir y conformar los materiales necesarios para satisfacer sus necesidades. En el presente trabajo se desarrolla un recorrido por las diferentes etapas por las que ha transitado la cultura humana y como ha contribuido en su desarrollo los elementos naturales y artificiales que los rodean; éstos son los materiales, con los que han conformado sus herramientas, su hábitat y sus relaciones con la naturaleza y sus congéneres. En la época actual conocer dichos materiales cobra particular importancia en cuanto a preservar y reconstruir la relación (en muchos casos dañada) con la naturaleza.

Palabras claves: Ingeniería, Tecnología, Naturaleza, Clasificación Materiales, Agrimensura. 


\section{Summary:}

Historically, the development and evolution of societies has been related to the ability of their members to produce and shape the materials necessary to satisfy their needs. In this paper, a journey through the different stages through which human culture has passed and how the natural and artificial elements that surround them have contributed to its development is developed; these are the materials with which they have formed their tools, their habitat and their relationships with nature and their fellow humans. In the current era, knowing these materials takes on particular importance in terms of preserving and rebuilding the relationship (in many cases damaged) with nature.

Keywords: Engineering, Technology, Nature, Materials Classification, Surveying

\section{Introducción: los materiales a lo largo de la historia}

Los materiales de construcción junto con la energía han servido al hombre para mejorar su calidad de vida. Los prehistoriadores han encontrado útil clasificar las primeras civilizaciones a partir de algunos materiales usados: Edad de Piedra, Edad de Cobre, Edad de Bronce, Edad de Hierro. Esta secuencia parece universal en todas las áreas, ya que el uso del hierro requiere una tecnología más avanzada que la producción de bronce, que a su vez requiere mayor tecnificación que el uso de la piedra.

En la siguiente gráfica (Imagen 1) podemos ver de manera simplificada y concomitantemente la utilización de los materiales a lo largo de la historia del hombre.

En la parte superior, el primer grupo que se observa son los Metales.

En el año 10.00o a.C. en este grupo, se utilizaban mayoritariamente metales preciosos como oro y plata. Estos eran los únicos que aparecían en la naturaleza en estado desoxidado. A medida que las técnicas evolucionaron, en el año 5.00o a.C. se incorpora la utilización del cobre, sobre todo para la construcción de herramientas rudimentarias. A continuación, se comenzó a trabajar con aleaciones del cobre 


\section{EVOLUCIÓN HISTÓRICA DE LOS MATERIALES}

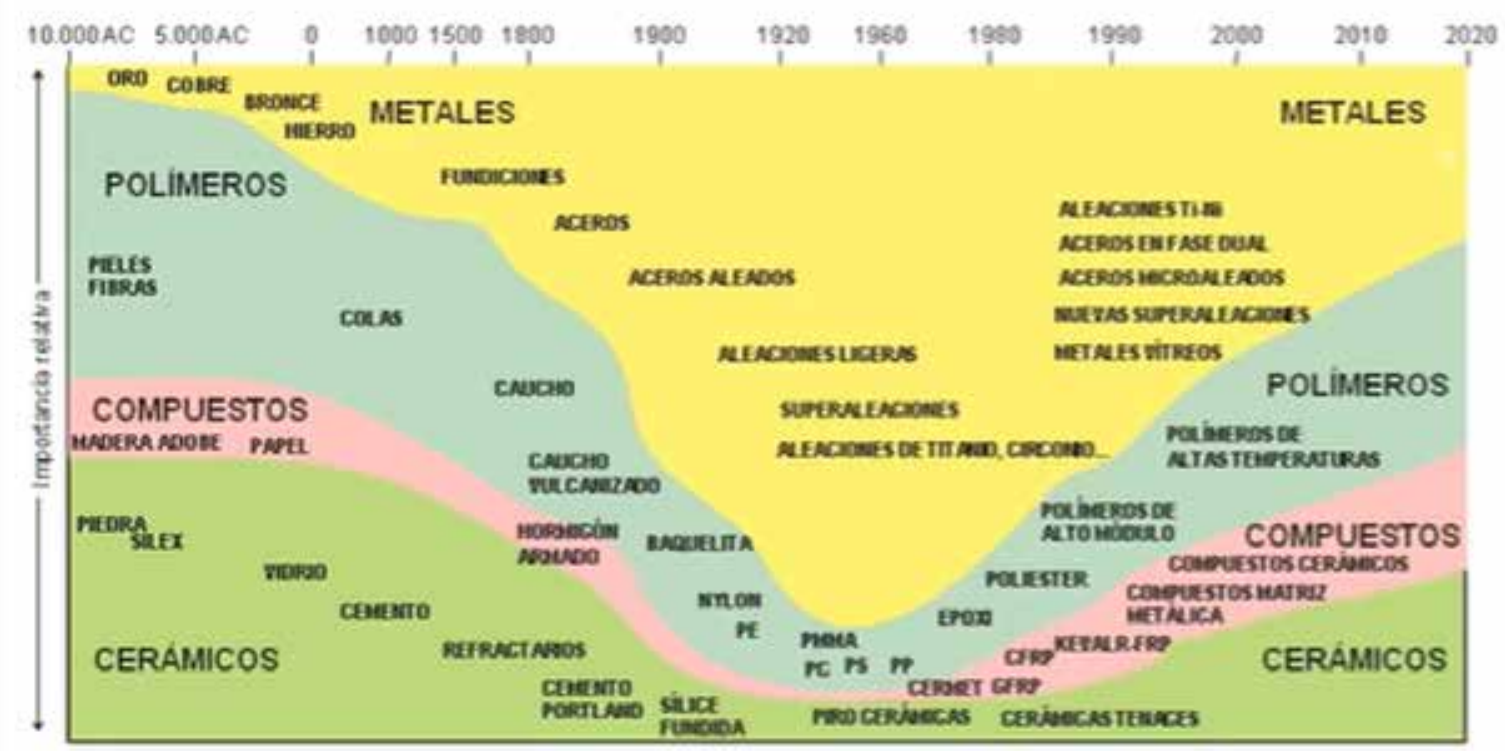

Imagen 1: Ayer, hoy y mañana de los materiales. Universidad complutense de Madrid. 2017.

y estaño, dando como resultado el bronce y se trabaja el hierro. Y más adelante a mediados del siglo XVI aparecieron las fundiciones con aleaciones de hierro. Para continuar en el siglo XIX cuando aparece el Acero, que es un material metálico que presenta una mayor resistencia mecánica que las fundiciones, para dar lugar ya más cercano a nuestros días, a los Aceros Aleados.

En el primer cuarto del siglo XX aparecieron las Aleaciones ligeras, aleaciones de Aluminio, que son ligeros y de baja densidad. Posteriormente se comenzaron a utilizar Súper - Aleaciones, que tienen como base al cobalto, níquel, titanio o circonio.

Hasta entonces la utilización de metales había crecido casi linealmente, y a partir de la década del 60 del siglo pasado, esta comenzó a disminuir debido al crecimiento en la utilización de otros materiales.

En los últimos años, se comenzó a utilizar aleaciones titanio - níquel, aceros micro - aleados, súper aleaciones de cobalto y metales amorfos.

El siguiente grupo de materiales en el gráfico es el de los Polímeros. 
Para empezar en el año 10.000 a.C. se utilizaban solamente pieles y fibras. Recién aparecieron las colas en el año 500 aproximadamente, que provenían principalmente de la pezuña de burro.

En los siglos XVI y XVII comenzó a utilizarse el caucho natural que provenía de una planta; más adelante se descubrió el caucho vulcanizado que es el que se utiliza en los neumáticos.

En 1909 se sintetizó el primer polímero sintético llamado Baquelita. A continuación, comenzó una escalada de polímeros sintéticos que dio lugar a la aparición del Nylon, el Polietileno, el Policarbonato, el Metacrilato, el Polipropileno, la Resina Epoxi y el Poliéster.

Estos plásticos comunes dieron lugar a plásticos con mayores prestaciones, como polímeros con un alto módulo de elasticidad y más adelante a polímeros resistentes a altas temperaturas.

\section{El tercer grupo de Materiales, es el de los materiales Compuestos.}

En los años 10.000 a.C. los compuestos más utilizados eran la Madera y el Adobe. Y más adelante aparece el Papel como sustitución del papiro.

A partir del año 1.00o se comenzó a utilizar el Cemento y posteriormente el Hormigón Armado que es un compuesto de Hormigón y estructura de Metal. Entre las décadas del 20 y 60, del siglo XX, apareció el Cermet, que es una mezcla de Metal y Cerámico.

Finalmente, tenemos los materiales compuestos incorporados recientemente, siendo los más conocidos la Fibra de Vidrio, Fibra de Carbono, Fibra Kevlar y se están investigando compuestos de matriz metálica y compuestos cerámicos.

El último grupo que consideramos en esta clasificación es el de los Cerámicos. 
En los primeros tiempos se utilizaba la Piedra como elemento constructor principal y el Sílex para la fabricación de herramientas. Más adelante, en el año 100 a.C., surgió el Vidrio y luego el Cemento. También en esta evolución aparecieron los Cerámicos Refractarios que soportan altas temperaturas.

Entre 1800 y 1980 se destaca la aparición del Cemento Portland, Sílice Fundida, Piro - Cerámicas y Cerámicas Tenaces.

En otro análisis atemporal según Pasman, podemos catalogar a los materiales de acuerdo a su Origen, así éstos pueden ser de procedencia Mineral, Vegetal y Complementarios.

\section{Clasificación seqún Pasman}

\section{Origen Mineral}

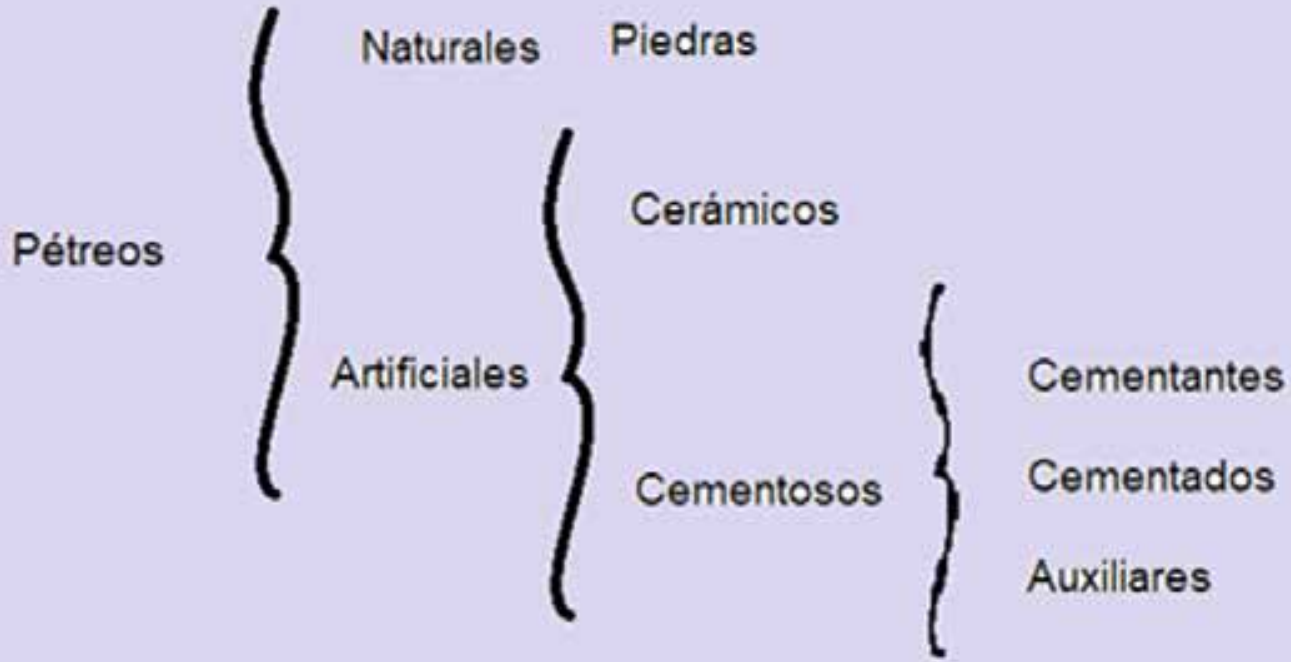

Bituminosos

Metálicos 


\section{Materiales de Origen Mineral Pétreos Naturales}

Pétreos: son las piedras naturales, pueden presentarse en forma de bloques, losetas o gránulos. Por ejemplo, el Mármol la Pizarra o la Arena. Éstos a su vez se clasifican en: Naturales: donde podemos encontrar a las Piedras, que constituyen uno de los materiales de construcción más antiguos. Se extraen de las canteras y posteriormente se labran, hasta que se consigue la forma, el tamaño y el acabado que se necesite. Suelen clasificarse según su origen en tres grandes categorías: Rocas Magmáticas o Ígneas, Rocas Sedimentarias y Rocas Metamórficas.

Las más empleadas en la construcción son: el Granito: que es una roca de origen magmático, dura, densa y resistente a los esfuerzos de compresión y a las condiciones del medio ambiente. Se pueden tallar y pulir, por lo que se consigue que su superficie adquiera un brillo intenso; los hay en distintos colores. Se emplean en la construcción de muros, pavimentos y encimeras.

Las piedras Calizas: son rocas de origen sedimentario que se han formado por la acumulación de sedimentos ricos en carbonato de calcio (huesos, caparazones, etc.). Se emplean en la construcción de muros y en la fabricación de cemento. Se tallan con facilidad y tienen gran porosidad. Y están las Areniscas: que son rocas sedimentarias de texturas granulada, formadas por arena unida con cementos naturales. Las hay de distintos tipos (sílice, caliza, arcilla, etc.), con diferentes resistencias, durezas y colores.

Tenemos las Pizarras: que son rocas metamórficas, las cuales pueden encontrarse en una gran variedad de colores, tonalidades y dibujos. Al igual que los granitos, se pueden tallar y pulir. Ofrecen gran resistencia a la comprensión, tienen gran densidad y mucha dureza; pero son sensibles a la acción de los ácidos. Se las utiliza para revestimientos de paredes y suelos.

\section{Materiales de Origen Mineral Pétreos Artificiales}

En este grupo, los materiales a su vez se sub clasifican en Cerámicos y Cementosos, y dentro éstos últimos encontramos a los Cementantes, Cementados y Auxiliares. 
Los Cerámicos: éste material tiene como característica en común que están compuestos por minerales, que se obtienen a partir de la mezcla de arcilla, feldespato y arena; esto explica los cambios en sus propiedades durante el proceso de elaboración, ya que se moldea en frío. La arcilla: es plástica y moldeable cuando el grano es muy fino y está húmeda. Cuando se seca se vuelve rígida y al cocerla a una temperatura elevada se vuelve vítrea. Por su parte el feldespato: reduce la temperatura necesaria para cocer la cerámica porque actúa de fundente; y la arena: actúa como relleno.

Se pueden añadir otras sustancias para aumentar la resistencia de la cerámica ante el calor, así se obtiene la cerámica refractaria; son materiales muy duros resistentes a las elevadas temperaturas y fáciles de moldear. Las baldosas, azulejos y la loza sanitaria se fabrica a partir de arcillas especiales a las que se les aplica un tratamiento de vidriado o esmaltado.

El otro grupo lo constituyen los materiales Cementosos, que a su vez se subdividen en: Cementantes, Cementados y Auxiliares.

Los materiales Cementantes: se denominan así a aquellos que por sí solos o en combinación con otros, son capaces de formar productos de hidratación, como hidratos de silicato de calcio, hidratos de aluminosilicatos, o también hidratos de aluminosilicatos de calcio. Se utilizan para complementar al cemento ya que pueden potenciar las propiedades del mismo, o en determinados casos, suplir algunas deficiencias de este para cumplir con las especificaciones de algún proyecto. Dentro de este grupo están las Cales, Cementos y Yesos.

Por su lado dentro de los materiales Cementados, podemos encontrar: los áridos: que están conformados por los materiales de construcción granulado; y que a su vez pueden ser naturales, artificiales y reciclados. Dentro de ellos y de acuerdo a su tamaño podemos considerar, las arenas: las cuales proceden de la desintegración natural o de la trituración de las rocas. Éstas son partículas cuyo diámetro no supera los cinco milímetros. La grava: comprende fragmentos de rocas cuyo diámetro es superior a los cinco milímetros, pero inferior a los cinco centímetros; y se usan en la elaboración de morteros. El canto rodado: son fragmentos de roca 
de tamaño comprendido entre los cinco y los veinticinco centímetros, que han sido transportados por el agua o el hielo, por lo que su superficie suele ser lisa y su forma redondeada. Resultan muy útiles por la facilidad con la que se adhieren al hormigón. Se usan como material de relleno para drenajes y en pavimentos.

Y en tercer lugar dentro del grupo nos quedan los materiales Auxiliares: constituidos por aquellos elementos que es posible agregarlos a los Cementantes, y por ello se los llama también agregados. Contribuyen a la resistencia del mortero y facilitan el fragüe; pueden ser finos o gruesos. Dentro de este grupo se encuentras los Morteros, Hormigón, Aglomerantes de Yeso, Cemento y Cal.

También debemos considerar los Materiales de Origen Mineral Bituminosos: que son sustancias de color negro, sólidas o viscosas, dúctiles que se ablandan por el calor y comprenden a aquellos cuyo origen son los crudos petrolíferos, como también a los obtenidos por la destilación destructiva de sustancias de origen carbonoso. Si son puros se llaman Betún, y cuando impregnan calizas, arcillas, pizarras, etc. se denominan rocas asfálticas. Dentro de este grupo se encuentran el betún, asfalto, brea y alquitrán.

Y por último siguiendo esta clasificación tenemos los Materiales de Origen Mineral Metálicos: aquellos que se caracterizan por compartir las siguientes propiedades: son buenos conductores del calor y la electricidad, poseen alta densidad, reflejan la luz, y son sólidos a temperaturas normales (excepto el mercurio). Éstos metales se extraen de las rocas, y en el caso del Oro, la Plata y el Cobre ya fueron utilizados desde la prehistoria y hoy son muy importantes en la tecnología. A los materiales metálicos cuyo componente principal es el hierro se los llama ferrosos, y el resto se llaman no ferrosos. Dentro de este grupo se encuentran: el Hierro, Acero y Fundición, Cobre, Zinc, Aluminio y Níquel, entre otros.

Siguiendo con la clasificación de Pasman respecto del origen de los materiales, debemos considerar los que son producto de la naturaleza y los materiales complementarios. Materiales de Origen Vegetal: se obtienen directamente de árboles, plantas y arbustos. Entre ellos tenemos la madera: que se obtiene del tronco de los árboles, y se caracteriza por su resistencia y abundancia en el medio ambiente. 


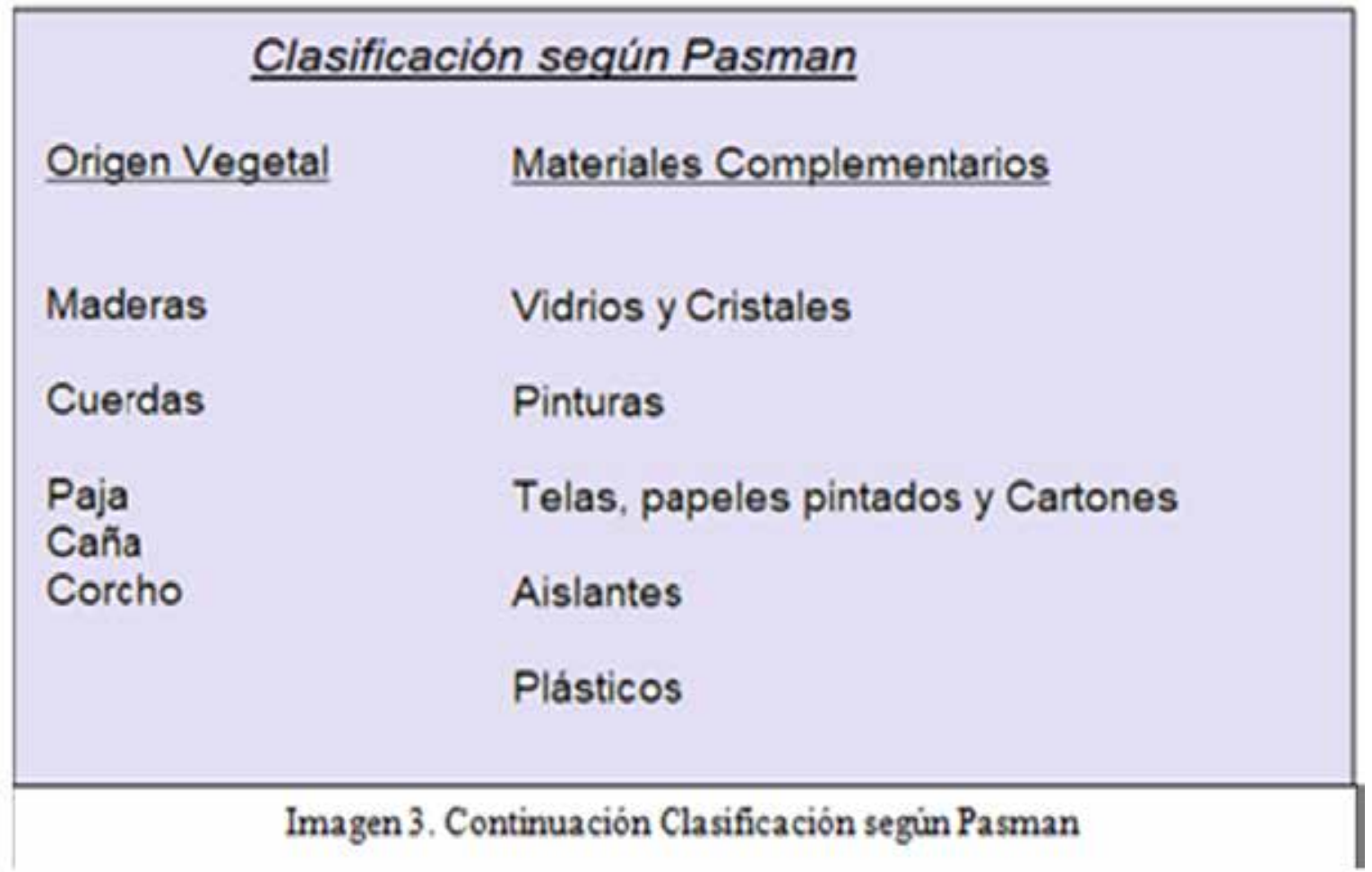

Otro es el caucho: material que surge de la savia de algunas plantas tropicales (Hevea Brasiliensis), que presentan una consistencia lechosa, el látex. Es normalmente empleado para elaborar neumáticos y artículos impermeables. Posee una gran ventaja, en cuanto a su elasticidad y resistencia a todo producto con propiedades ácidas. Dentro de esta clasificación también se encuentran las Cañas, Cuerda, Paja y Corcho.

Y el otro grupo comprende a los Materiales Complementarios: dentro de los cuales debemos considerar una nueva sub - clasificación: los vidrios y cristales; las pinturas, también el grupo de telas, papeles pintados y cartones; los materiales aislantes y por último los plásticos.

Los Vidrios y Cristales: se moldean en caliente, son transparentes, duros y resistentes a la corrosión; también son buenos aislantes de la electricidad. Son muy frágiles y aguantan mejor los esfuerzos de compresión que los de tracción. El vidrio se obtiene a partir de la fusión de arena, álcali y óxidos metálicos; después se le da forma antes de enfriarse. Se lo emplea en ventanas, recubrimientos externos y como aislante. Dentro de este grupo están el vidrio soplado, hilado y colado, lana de vidrio y vidrio de seguridad, entre otros. 
Las Pinturas: dentro de este grupo encontramos las pinturas vinílicas, el esmalte sintético, vinílicas de esmalte al agua, anti humedad, al aceite y barnices entre otros.

Las Pinturas Vinílicas: son aquellas que utilizan resinas vinílicas y sintéticas termoplásticas, para aglutinar sus componentes. Son aptas para aplicar en exteriores por su resistencia a la intemperie. Las Pinturas Plásticas: a su vez están compuestas por resinas vinílicas o acrílicas; y se utilizan sobre superficies de madera o hierro. Las Pinturas Anti - humedad: como su nombre lo sugiere se utilizan en lugares que están expuestos a mucha humedad, porque crean una barrera de impermeabilización. Y luego están los Barnices: que imitan el color de la madera con acabados transparentes.

\section{Telas, papeles pintados y cartones}

Materiales Aislantes: dentro de éstos de acuerdo al tipo de protección que brindan podemos considerar los hidrófugos, antitérmicos, antisónicos e ignífugos.

Materiales con efecto Hidrófugo: el aislamiento de éste tipo actúa como barrera contra la humedad para evitar su ingreso o filtración por los distintos elementos que constituyen el edificio.

Materiales con acción Antitérmica: ellos separan un espacio climatizado del exterior, o de otros espacios para reducir la transmisión de calor entre ellos. También se utiliza para reducir la transmisión de calor desde conducciones que transportan fluidos a distintas temperaturas. Aunque existen muchos aislantes específicos, se pueden diferenciar varios tipos de materiales: los Minerales: están formado por un entrelazado de filamentos de materiales pétreos que forman un tejido que mantiene entre ellos aire en estado inmóvil y ofrecen elevados niveles de protección frente al calor, el ruido y el fuego. Los Celulares: son materiales que se conforman en celdas cerradas o abiertas, por lo general formando tableros rígidos o flexibles, aunque también se pueden conformar in situ por proyección o riego. Los más usados son el Poliuretano y el Poliestireno Expandido. Los Granulares: formados por pequeñas partículas de materiales inorgánicos aglomeradas en formas 
prefabricadas o utilizadas sueltas, como la Perlita y la Vermiculita. Y los Orgánicos: conformados por materiales de ésta naturaleza pero comprimidos, entre los que destaca diversos tipos de planchas de Corcho Aglomerado.

Materiales con efecto Anti - Sónico: no todos los materiales reflejan las ondas sonoras con la misma intensidad. Un material poroso que contenga muchas moléculas de aire absorberá los sonidos que inciden en el con mucha mayor proporción que un material más compacto. En las radios o salas de teatro se suelen usar maderas perforadas o goma espuma en las paredes como aislantes del sonido.

Materiales con acción Ignífuga: son aquellos materiales que ofrecen resistencia el fuego y que evitan su propagación. Dentro de los materiales ignífugos tenemos a las espumas aislantes para paredes y suelos, placas de fibrosilicato, pintura intumescente, placas de lana roca, entre otros.

Materiales Plásticos: éste último grupo es uno de los materiales más utilizados en la construcción, ya que es ligero, barato y muy versátil. Dentro del mismo se encuentra una gran y variada oferta, donde podemos considerar: el Acrílico: que se utiliza como material rígido, en ocasiones para sustituir el vidrio, por ejemplo en las mamparas. El Polietileno: formada por una membrana plástica que se utiliza como embalaje y para aislar algunos metales y cables. Se utiliza en la construcción para aislar el edificio del terreno en losas. PVC: es la variedad de plástico más utilizada en la construcción; su uso va desde suelos de vinilo, revestimientos impermeables, tubos de fontanería, tubos de saneamiento, etc. El Polipropileno: se suele utilizar para realizar las cajas de los distintos puntos eléctricos, también en tubos de fontanería, instalaciones de climatización, etc. Y por otro lado el Poliestireno: que se utiliza sobre todo para aislamiento en su versión de espuma, también se usa para embalajes y elementos de decoración como molduras de techos. El Acetato de Polivinilo: se suele encontrar en emulsiones para pinturas, en los acabados para suelos y multitud de adhesivos y colas.

El Nylon: se utiliza más que nada en elementos decorativos para el interior como alfombras o barras para cortinas, entre otros. 
La Melamina: se utiliza sobre todo para los revestimientos de tableros aglomerados de muebles y mesas de trabajo, también se puede utilizar para hacer mamparas de baño, cabinas sanitarias, etc. El Poliuretano: que se utiliza en todo tipo de pinturas y barnices y también como material aislante. Y por último tenemos la Resina Epoxi: que se utiliza sobre todo como colas, adhesivos y también como terminaciones y revestimientos.

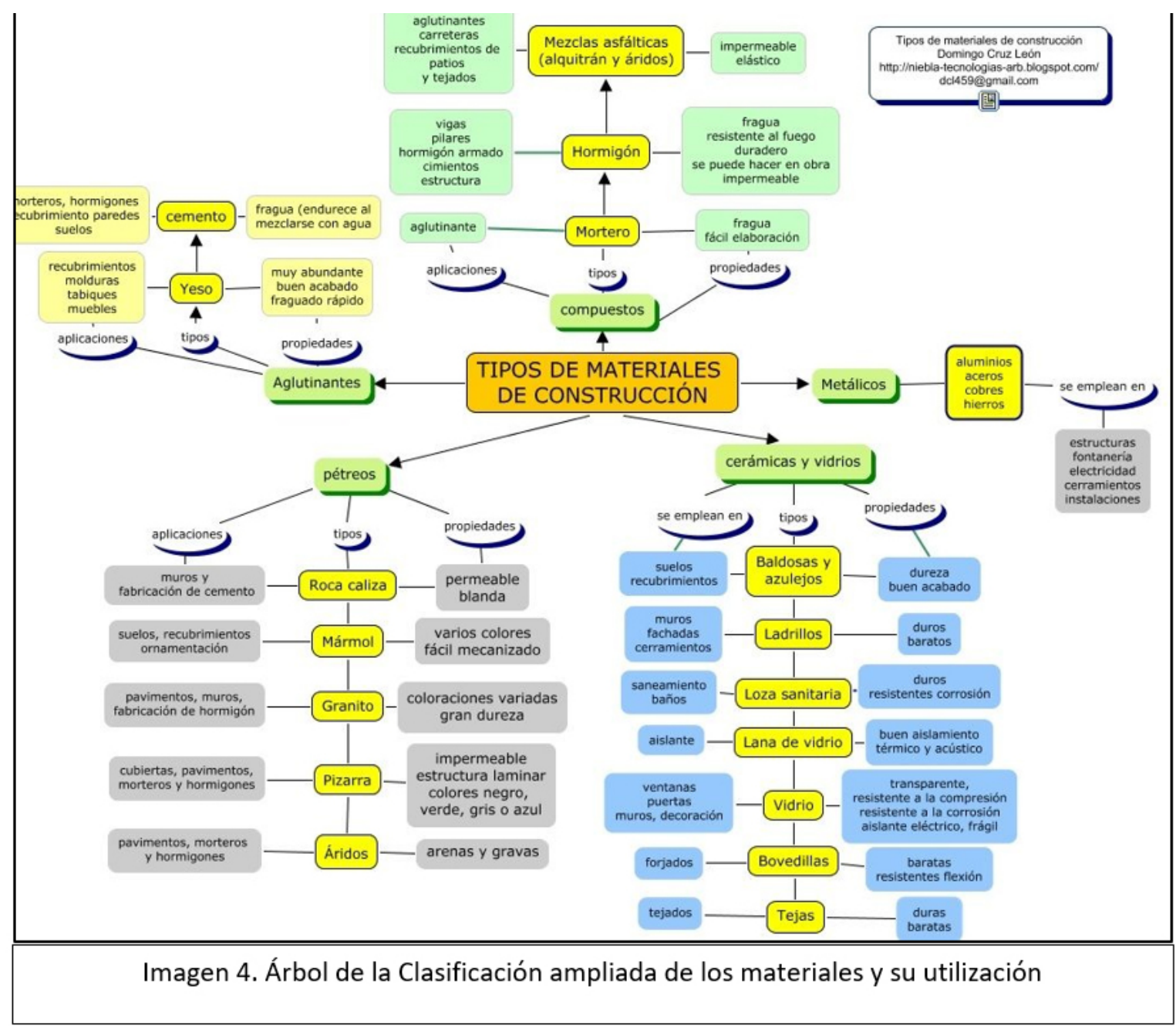

De acuerdo a lo visto sobre la tendencia en la evolución de los Materiales, se observa que existen características que son buscadas para un mejor uso y aprovechamiento de los mismos. También es oportuno considerar la creación y búsqueda de nuevos materiales a lo largo del tiempo, donde existen algunas tendencias respecto a sus propiedades y características, siendo las más importantes: 
- Reducción en el costo energético

- Reducción de peso

- Incremento de resistencia

- Reducción de contaminación

- Biodegradabilidad

Un ejemplo de ello pueden ser los nuevos vidrios fotovoltaicos que incorporan semiconductores transparentes basados en celdas fotovoltaicas como sistema Fotovoltaico Integrado en Edificios, o Sistema BIPV (Building Integrated Photovoltaics) integración de un elemento fotovoltaico en el propio edificio como una parte de él. Se trata de la integración de módulos fotovoltaicos en la envolvente del edificio, principalmente en cubierta o fachada, para cumplir una doble función; como material envolvente de construcción sobre el edificio convencional y como un sistema generador de energía. De esta forma se puede tener una ventana, abertura o acristalamiento, que además de permitir el paso de la luz, genera electricidad.

\section{Conclusiones}

Los distintos materiales fueron evolucionando a lo largo de la historia del hombre de acuerdo a las necesidades de cada época y la tecnología alcanzada. Un claro ejemplo de esto es el aumento lineal en la historia, de la utilización de metales hasta su auge en la mitad del siglo pasado; que justamente coincide con los tiempos de revolución industrial y guerras mundiales. A su vez el uso de metales disminuyó linealmente hasta nuestros días por la aparición de nuevos materiales que los han reemplazado.

En la actualidad es un común denominador en la construcción de cualquier edificio que intervengan materiales pertenecientes a todos los grupos y orígenes. Sin embargo algunos materiales se volvieron fundamentales en la construcción del hábitat humano, como es el caso de los plásticos, que se utiliza en casi todos los ámbitos de la edificación.

Como vimos, el desarrollo de la tecnología permite alterar las propiedades de los materiales de acuerdo a las necesidades actuales. La tendencia en los últimos años 
es generar y emplear materiales amigables con el medio ambiente, que los mismos reduzcan la contaminación y sean biodegradables.

Por ello es importante que se siga profundizando en su estudio, se difunda y se genere conciencia sobre materiales ecológicos, promoviendo la utilización de los mismos y considerar también la sinergia con los materiales relacionados con las energías renovables.

\section{Bibliografía:}

Chandias M. E. Campos J. M. Computos y Presupuestos: manual para la construcción de Edificios con computación aplicada. Librería y Editorial Alsina. Buenos Aires. (2015)

Construmatica; https://www.construmatica.com/construpedia/

Corvalán, R. E. De Thales de Mileto a la Nueva alianza de Prigogine y Stengers: Línea de tiempo de la Filosofía de la ciencia y la naturaleza. ACADEMO (Asunción) 5(1):61-72. (2018).

Crespo Escobar S. Materiales de Construcción para Edificaciones y Obra Civil. Editorial:, ECU . Alicante - España. (2010).

Distintos tipos de plásticos usados en construcción. https://vilssa.com/

Fernandez Sanchez P.; Pastor Caño I., Hoy, ayer y mañana de los materiales. Universidad Complutense de Madrid, Universidad Politécnica de Madrid. (2017).

Kottas Dimitri, Materiales para la construcción. Pluton ediciones. Barcelona - España. (2016)

Pasman, M. F.; Materiales de Construcción. Cesarini Hermanos editores, octava edición. Buenos aires. (1997)

Saavedra Romero G., Materiales sintéticos de Construcción, https://es.scribd.com/ document/477329105/MATERIALES-SINTETICOS

Tres cientos sesenta en concreto, comunidad de ideas sobre la industria y el mundo de la construcción https://www.36oenconcreto.com/blog/detalle/efectos-de-cementantes-suplementarios. 